\title{
EDIFICIO DE OCIO Y ESPECTÁCULOS. PLAZA DE TOROS EN MÓSTOLES, MADRID/ESPAÑA
}

\author{
(BUILDING DEVOTED TO ENTERTAINMENT AND LEISURE. BULLRING IN MÓSTOLES, \\ MADRID/SPAIN)
}

Javier Casado Terán; Mauro Barrientos González y Jesús Ramos Paños, Arquitectos

José Antonio Abellán, Aparejador

ESPAÑA

Fecha de recepción: 29-XII-96

\begin{abstract}
RESUMEN
Los edificios taurinos, tal como los hemos conocido, son el resultado de un largo proceso evolutivo, en el cual han existido momentos concretos de innovación y cambio. En España vivimos ahora el que, probablemente, deba ser uno de estos momentos.
\end{abstract}

Las plazas de toros llevan ya tiempo intentando abrirse a otros usos, lúdicos o culturales, en busca de una mayor rentabilidad de sus instalaciones. Hasta el momento, este proceso no ha generado modificaciones que permitan el desarrollo de las distintas funciones en las mejores condiciones posibles, sino que suele recurrirse a montajes temporales.

Este edificio plantea una solución a este problema, integrando en un mismo espacio, de forma estable, dos funciones de ocio distintas, la taurina y la teatral. Se sopesan los condicionantes derivados de cada una de ellas para obtener un resultado final capaz de conjugar la centralidad del coso con la frontalidad del auditorio.

El escenario, como elemento característico de la nueva función, busca su ubicación en un lugar visible y asequible como lo es una localidad más del tradicional graderio. Las localidades que ahora ocupa se trasladan al punto diametralmente opuesto, conservando siempre la continuidad circular, fundamental para el desarrollo del espectáculo taurino. Así, el graderio se desplaza para dar entrada al nuevo elemento, la escena, que usurpa su tradicional sentido horizontal.

Todo esto utilizando una arquitectura propia -y por lo tanto contemporánea- que propone una vía de evolución para el futuro desarrollo de nuestros cosos taurinos.

\section{SUMMARY}

The traditional image of the buildings for the "Fiesta" must be seen as a point in a long process of definition in which important changes and innovations have been assimilated. Nowadays, things that should derive in some of these changes are happening.

The "Plazas" are being used for purposes so different to those which motivated their construction as being the site for concerts or diferent kinds of shows.

What most defines the traditional building is the centrally focalizated definition of the seats that allows a clear vision from any point of the ruedo. Introducing a scene box meant extracting this focus from the "ruedo", so the optimal location should be found.

Traditionally, the public has taken an active role in the "corrida". Each one of the seats is a place to look from, but it becomes too a place to be seen. So this seemed like the natural location for the scene, that will remark this peculiar relationship between the two main parts of the building,, the rows of seats and the "ruedo". Obviously, the total amount of seats should be kept, so those to be invaded by the scene were displaced to the diametrally opposite point. What we, finaly, have, is a traditional "ruedo" surrounded by a crown of seats with a varying width, where a scene box is placed.

This compromise between Tradition and Evolution is what we propose as a way to explore in the future evolution of the "Plazas". 


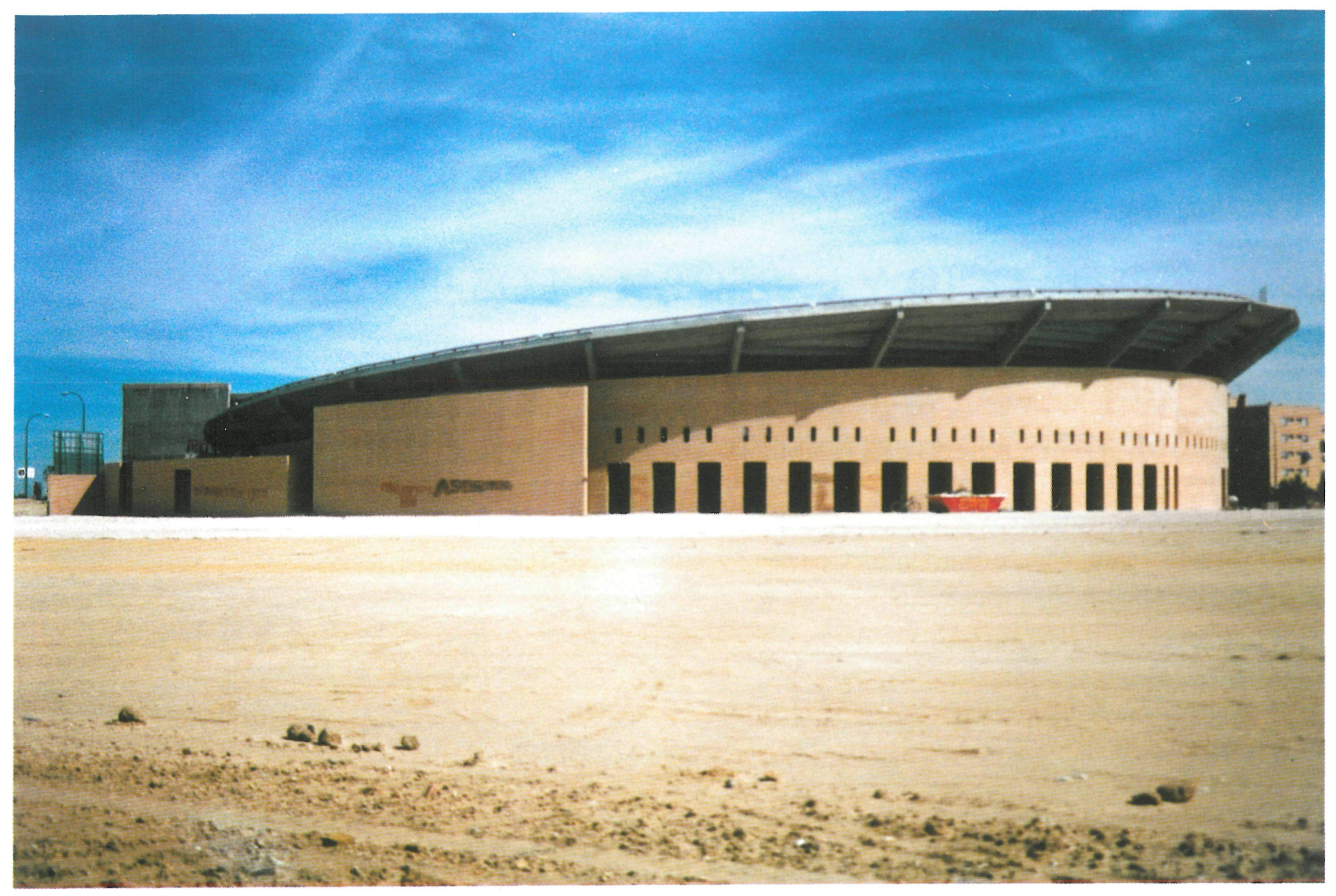

Vista de la plaza terminada.

\section{Condicionantes}

El Concurso de Ideas promovido por el Ayuntamiento de Móstoles planteaba un problema interesante, pero cuya solución no resultaba, a priori, sencilla. Se proponía diseñar una Plaza de Toros, cuyo uso debería ser compatible con la realización de otro tipo de espectáculos y manifestaciones culturales (música, teatro...). Además, se sugería la posibilidad de hacerla cubierta.

Las bases recogían, de este modo, la tendencia actual a buscar una mayor rentabilidad de la inversión que supone un edificio de estas características, permitiendo su uso más allá del corto período de tiempo que supone una temporada taurina. Este uso combinado, que de hecho ya se da en gran parte de las plazas españolas, no se había traducido, hasta el momento, en cambio alguno en la configuración de los cosos, a pesar de que, a nuestro juicio, su perpetuación ha de entenderse como un cambio sustancial en la naturaleza de los espacios dedicados a la Fiesta.

Por tanto, abordamos el problema como el, por ahora, último de los momentos clave que a lo largo de la historia de esta tipología han generadó una evolución formal. Evolución que debía producirse desde el escrupuloso respeto a unas normas muy concretas, como son las recogidas tanto por el Reglamento Taurino como por el saber popular. Éramos conscientes del peso que tiene esta última normativa no escrita, planteándonos, como reto, conjugar la necesidad de Evolución con el respeto a la Tradición.

Otro condicionante que había de resultar de gran importancia en el desarrollo de la obra fue el carácter propagandístico que ésta iba a cobrar para el equipo de gobierno del Ayuntamiento, que se traduciría en un plazo de ejecución muy estricto, de cara a poder cortar la cinta correspondiente en el momento más rentable políticamente.

\section{Solución formal}

El principal problema formal que se presentaba, a la hora de conseguir conjugar las dos modalidades de uso del edificio, era la ubicación de un elemento como es el escenario, alterando lo menos posible la imagen, ya consolidada, del coso.

Consideramos que cualquier localidad de la grada cumplía ya esa doblefinalidad, de ver y ser visto, contribuyendo, en gran medida, al colorido del espectáculo, con lo cual resultaba inmediato transformar una de ellas en el propio 


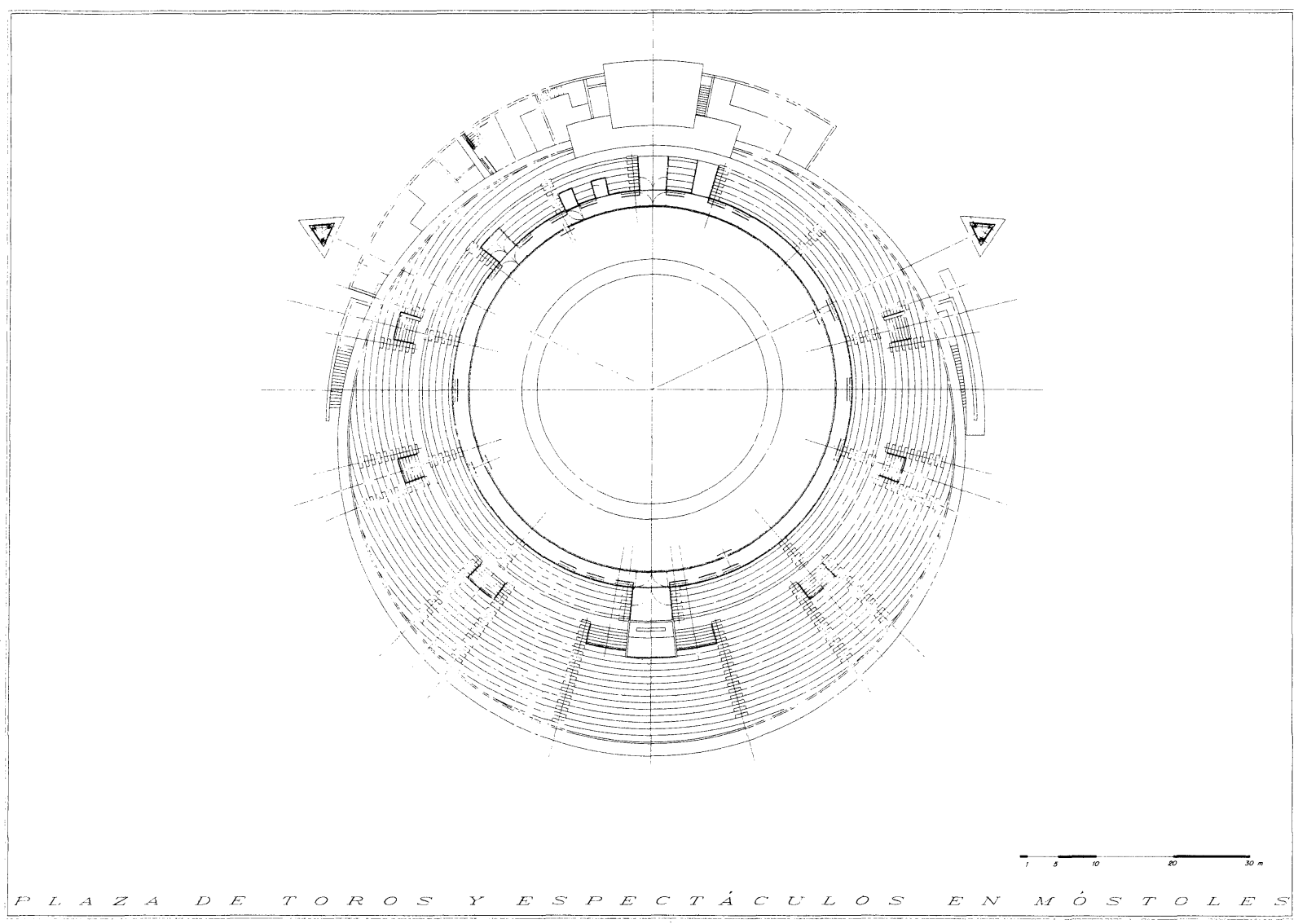

Planta de gradas.

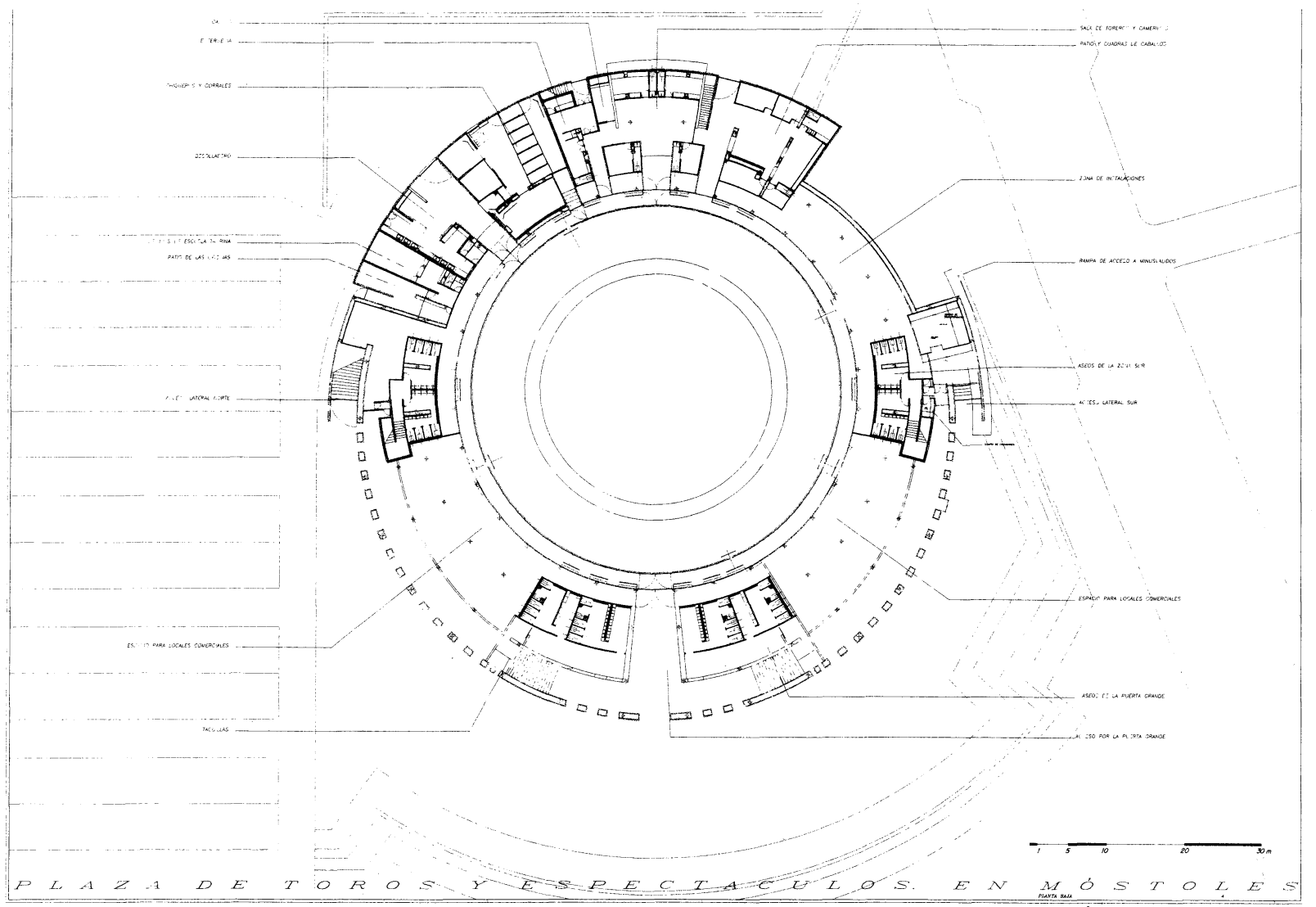




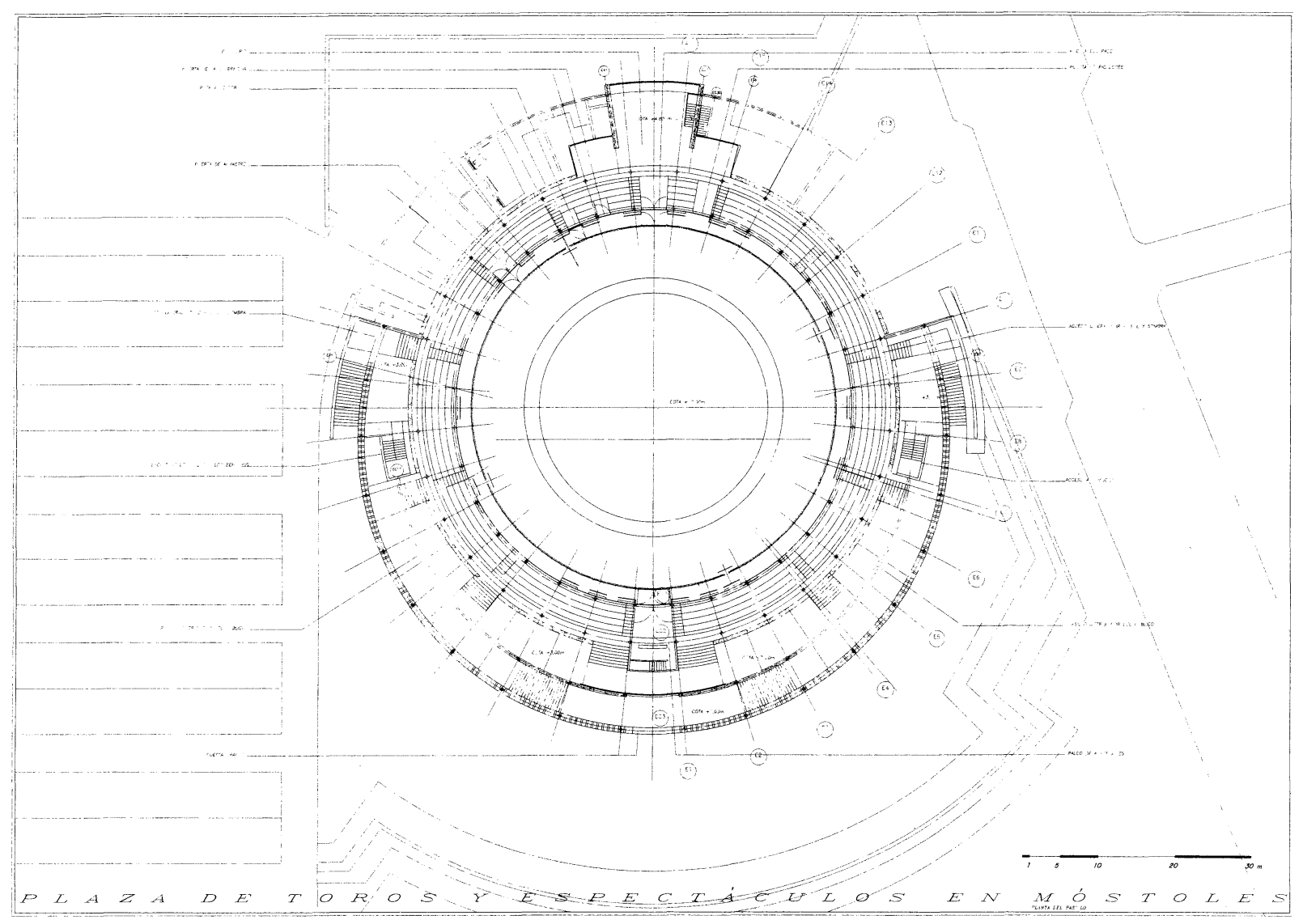

Planta del pasillo.

escenario. Pero éste debía tener la capacidad suficiente para acoger los espectáculos previstos, y era importante conseguir, al tiempo que la cercanía suficiente al público que, en pie, ocuparía el ruedo, no alterar la continuidad del anillo que da su forma tan característica a la planta de una Plaza de Toros.

El escenario se formaliza, así, por medio de una caja de hormigón que coloniza una de las localidades para reservar su uso. Esta situación provoca el traslado de las localidades vecinas al punto diametralmente opuesto de la plaza, de manera que el graderío tiene una altura mínima en la zona del escenario y máxima en la correspondiente al palco presidencial, según se aprecia en la sección. Esta imagen peculiar, que recuerda un platillo inclinado, resulta, por tanto, de superponer al esquema de focalidad "central" que caracteriza al espacio tradicional de una Plaza de Toros, la focalidad "lineal", propia de un Teatro.

De esta superposición se toman los elementos que resuelven la Arquitectura del edificio, el círculo inherente a esta tipología y el radio, que surge de manera inmediata al fijar un punto prioritario de la grada. Una serie de muros concéntricos definen los espacios y circulaciones; mientras que los distintos radios ordenan las funciones de la plaza. El único muro que, aun describiendo un círculo en planta, desplaza su centro, siempre dentro del eje principal del edificio, es el que define el alzado principal. El citado eje principal, que por ahora relaciona visualmente el escenario con la zona de mayor número de localidades, es, además, un eje fundamental del espectáculo taurino, ya que en él se ubican la Puerta del Paséillo y el Palco de la Presidencia (eje del paseíllo), que se ha hecho coincidir con el de la Puerta Grande.

Para resolver el problema de la orientación del edificio se tuvieron en cuenta dos elementos meteorológicos de gran importancia en el desarrollo de la lidia, como lo son el viento y el sol. Estudiadas las condiciones del lugar, se buscó que la "línea de sol y sombra" definiera la mayor cantidad posibledelocalidades resguardadas, aprovechando para ello la inclinación del graderío. De la misma manera, se resguardaba el ruedo de los vientos dominantes y su pernicioso efecto. La alineación de la puerta grande con el citado eje del paseíllo permitía que el eje fundamental del edificio se correspondiera con el futuro eje urbano previsto en el P.A.U. 


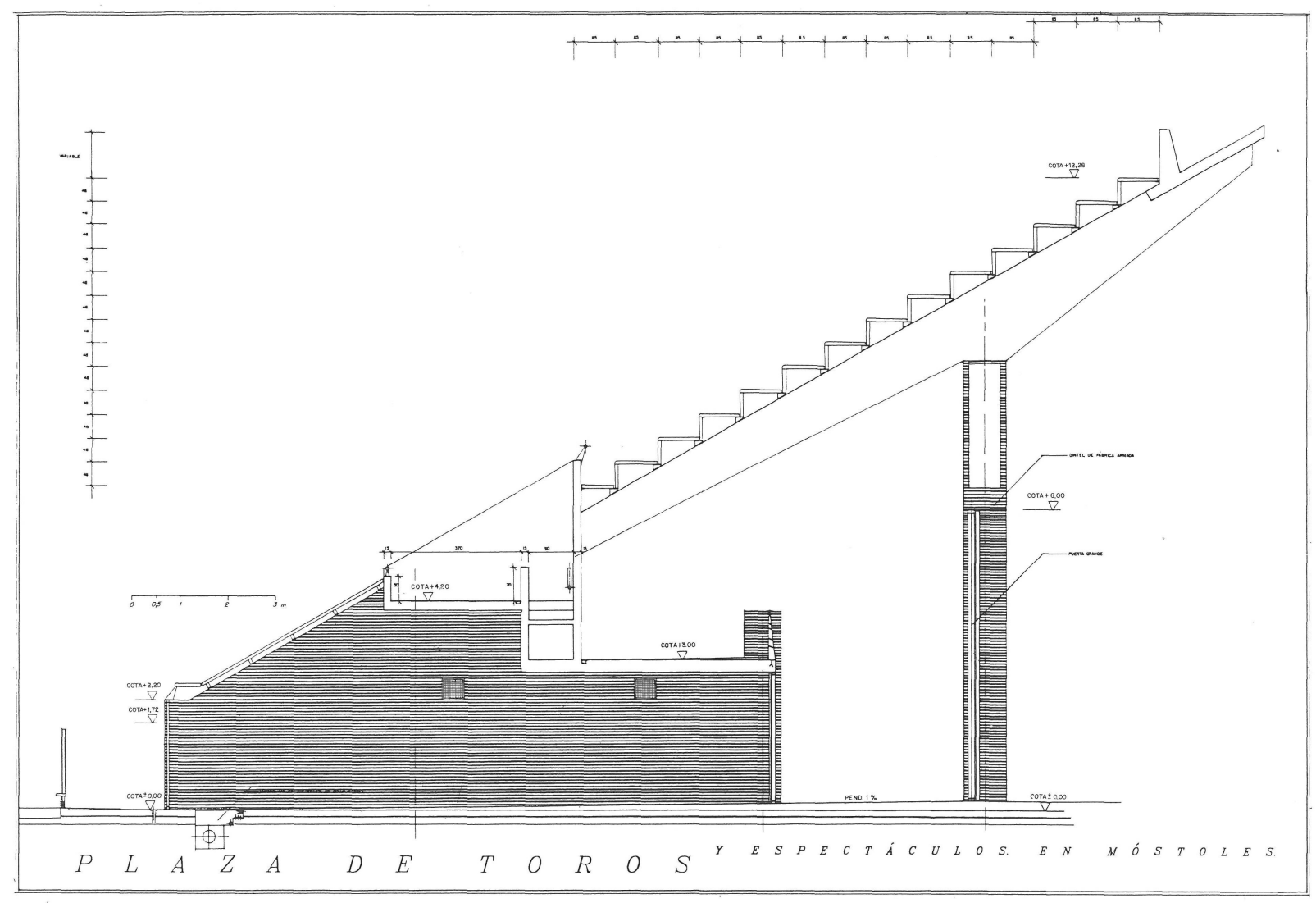

Sección por el eje de la Puerta Grande.

\section{Solución estructural}

En un edificio de estas características, la estructura no sólo debe cumplir su función portante, sino que desempeña un importante papel en la definición del espacio y la propia imagen final. Era importante manifestar los elementos constructivos de la plaza de manera singular, haciéndolos fácilmente identificables y comprensibles al público. Por estos motıvos, era fundamental conseguir el acuerdo entre la forma derivada de la función resolutiva del espacio y la óptima, debida al cálculo.

Los motivos que llevaron al empleo de una solución prefabricada fueron, fundamentalmente, la economía que se debía derivar del uso de elementos repetitivos y la premura de tiempo, debida a la necesidad de que la plaza pudiera acoger los primeros festejos en una fecha concreta.

Sin embargo, la definición arquitectónica del edificio determinó que los pórticos radiales, que se presentaban como la solución lógica, no fueran todos iguales, puesto que, como queda dicho, la anchura de la grada crece progresivamente entre el escenario y la zona de la tribuna.
Este crecimiento implica una mayor luz de las vigas, que, lógicamente, requieren un canto también mayor. Los pórticos presentan, además, un voladizo terminal, donde, aparte de compensar la luz mayor, contribuye a manifestar al exterior la propia naturaleza constructiva del edificio, y que también debe adaptarse a una solución de borde, especialmente compleja.

Uno de los primeros objetivos fue, por tanto, conseguir diseñar un sistema de prefabricación que permitiera el empleo de un solo molde, lo que se consiguió finalmente gracias a un intenso trabajo, en colaboración con los ingenieros de la empresa.

El diseño delas gradas presentaba, decara ala prefabricación, problemas similares a los de los pórticos, derivados también del encuentro de dos geometrías circulares desplazadas. Las gradas se resolvieron con piezas en forma de L, que hicieron las veces de asiento y respaldo. El carácter radial hace que las sucesivas gradas vayan aumentando su luzy, al no poderse aumentar el canto (altura del asiento), se optó por aumentar el ancho de la parte resistente de la grada (respaldo). Este aumento afectó, también, al apoyo horizontal de las gradas sobre los pórticos. 


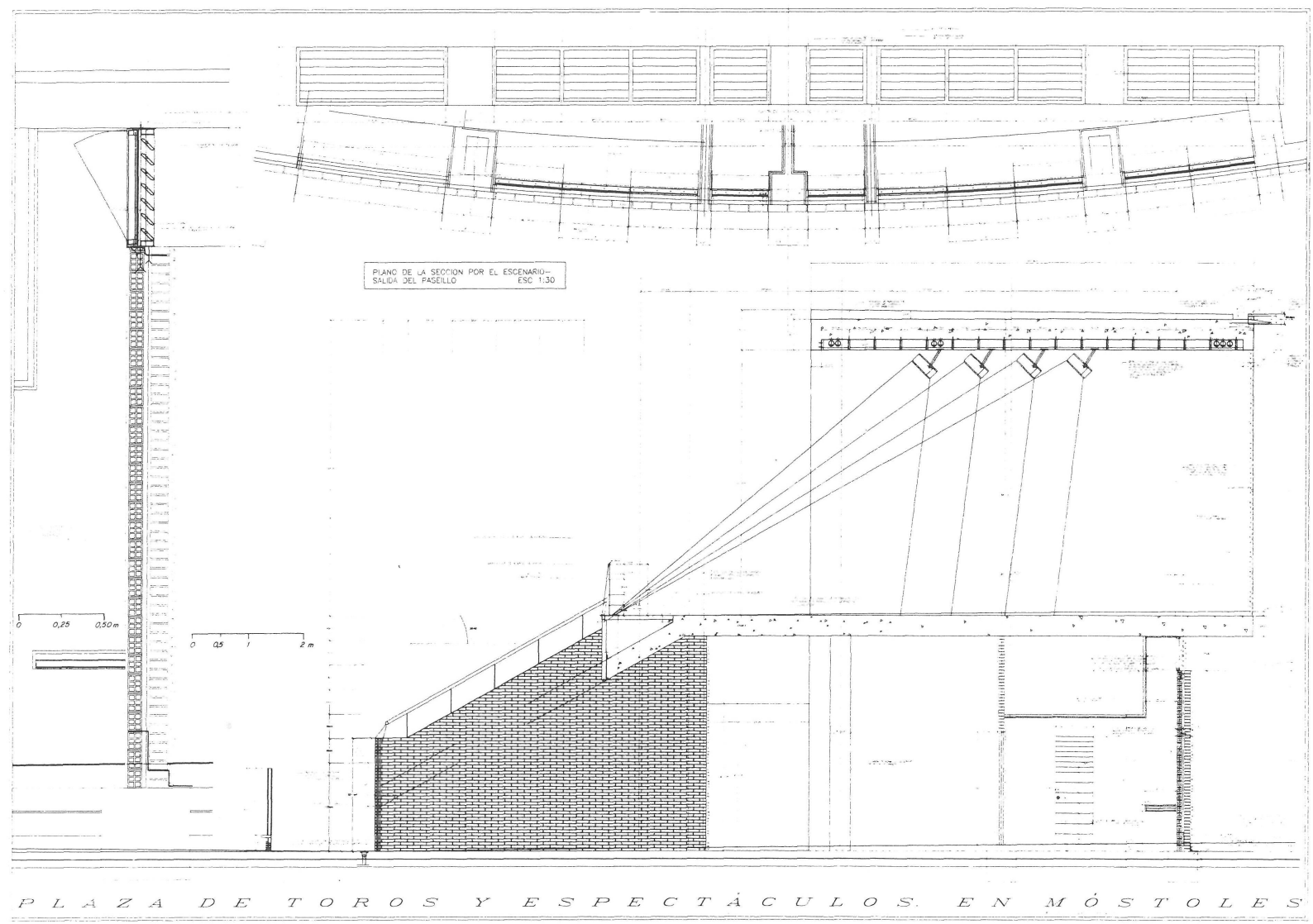

Sección por el escenario.

La configuración formal de las gradas se traduce en un anillo perimetral de geometría compleja, obtenido por la intersección del virtual cono invertido del graderío por un plano inclinado. Sobre este anillo elíptico, cuyos tramos tienen luces variables, han de irse apoyando las gradas más altas de los tendidos. Esta pieza, por tanto, tiene una función estructural importante, además de su valor espacial. El canto necesario para la pieza se absorbió en el necesario peto de protección. Estas consideraciones se complicaban, debido a que también variaba la posición espacial final de cada pieza y su relación con los pórticos $y$, por tanto, su forma de trabajo.

\section{Proceso constructivo}

Si algo caracterizó el proceso de construcción de la Plaza fue su rapidez. De hecho, la prensa local y la población de Móstoles acuñaron el apelativo de "el coso vertiginoso". Esta rapidez se consiguió gracias a un verdadero esfuerzo en la coordinación de las distintas fases del proceso, especialmente en la puesta en obra de los elementos prefabricados

El propio carácter radial del edificio facilitó, de forma sustancial, el replanteo de la cimentación, realizada in situ con zapatas de hormigón armado. Una vez realizada la cimentación se comenzó el proceso de ensamblaje de los pilares de los pórticos, mediante un sencillo sistema de vainas.

Cada pórtico, de dos tramos, está compuesto por tres pilares y dos tramos de viga, variable en su longitud, contando, el de mayor luz, con un voladizo que le compensa en parte. El montaje se realizó en dos direcciones, radial, de dentro hacia afuera, y circular, "desplegando" la plaza como si fuera un enormeabanico. Una vez colocados y fijados los dos primeros pilares de cada pórtico y mientras se colocaba el tercero, se procedió a fijar el primer tramo de viga. En cuanto un pórtico estaba completo y el siguiente comenzado, se empezaban a tender las gradas, consiguiéndose, de esta manera, simultanear la entrada en obra de los distintos oficios, de forma que, en determinados momentos, fue posible observar, a la vez, todas las fases del proceso.

Hay que destacar la importancia que tuvo el control intenso de la realización del muro exterior, por tratarse de ladrillo cara vista en desarrollo curvo. El remate del muro, obtenido por una sección inclinada de su superficie, se obtuvo, efectivamente, cortando los ladrillos necesarios, para obtener realmente la continuidad deseada.

El empleo de los materiales permite resaltar ese juego entre la tradición y la contemporaneidad. El predominio del ladrillo cara vista, color ocre, cálido, parte de esa 


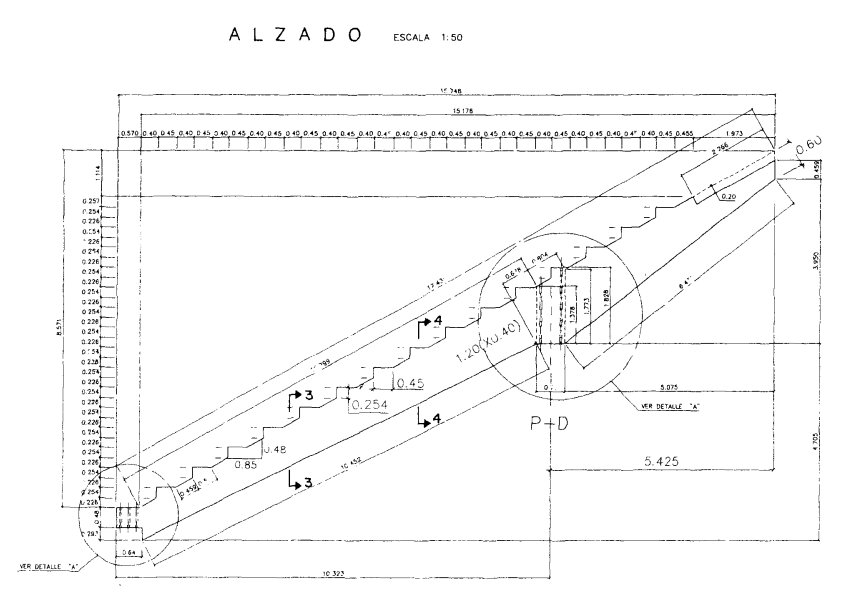

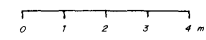

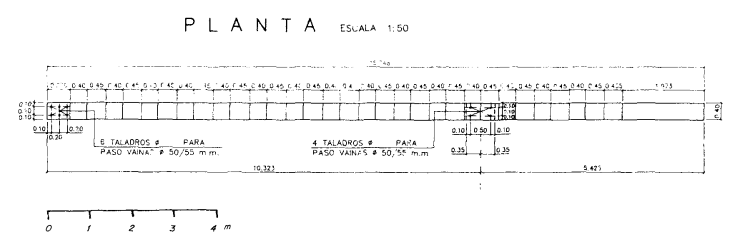

Estructura dintel (pórtico-1).
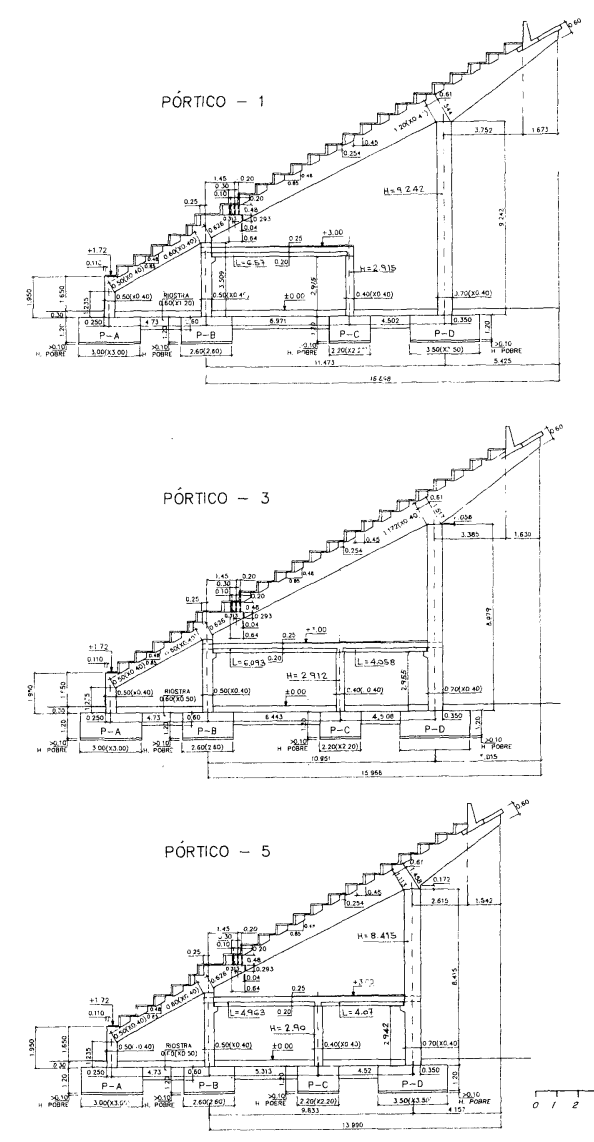

T.1
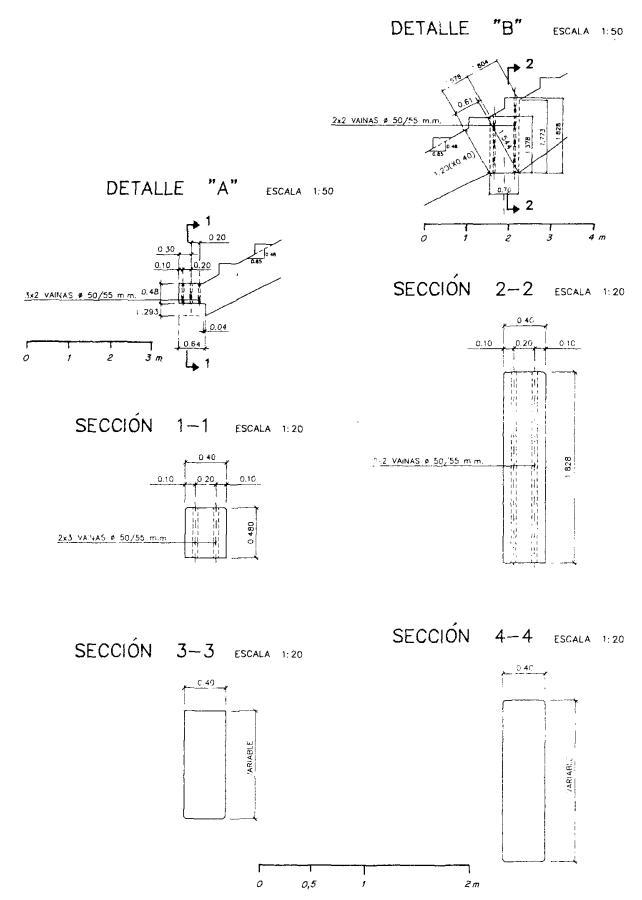

Estructura (pórticos la 6).
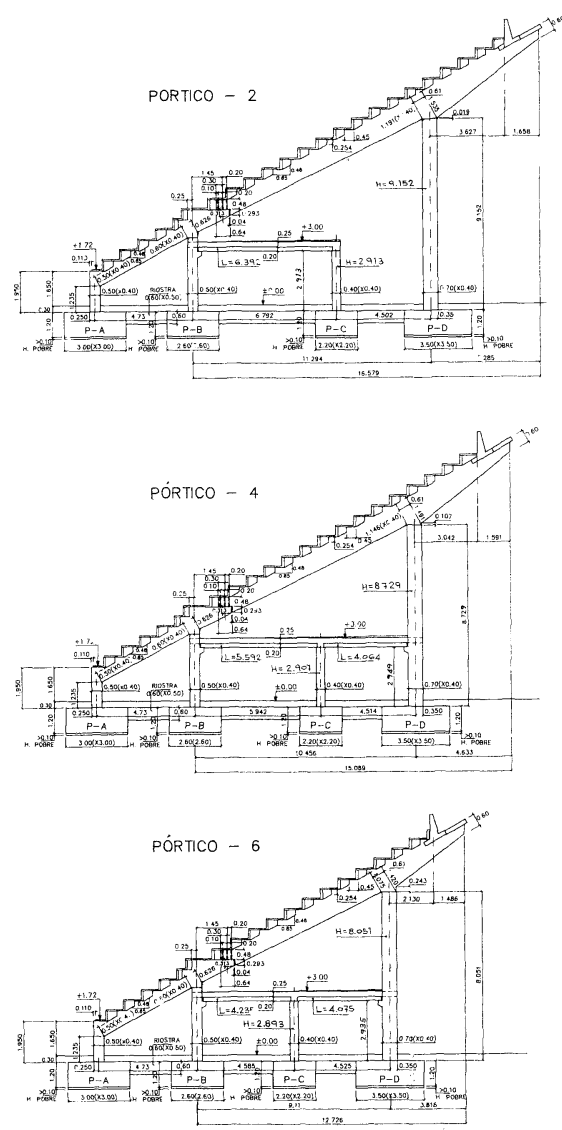

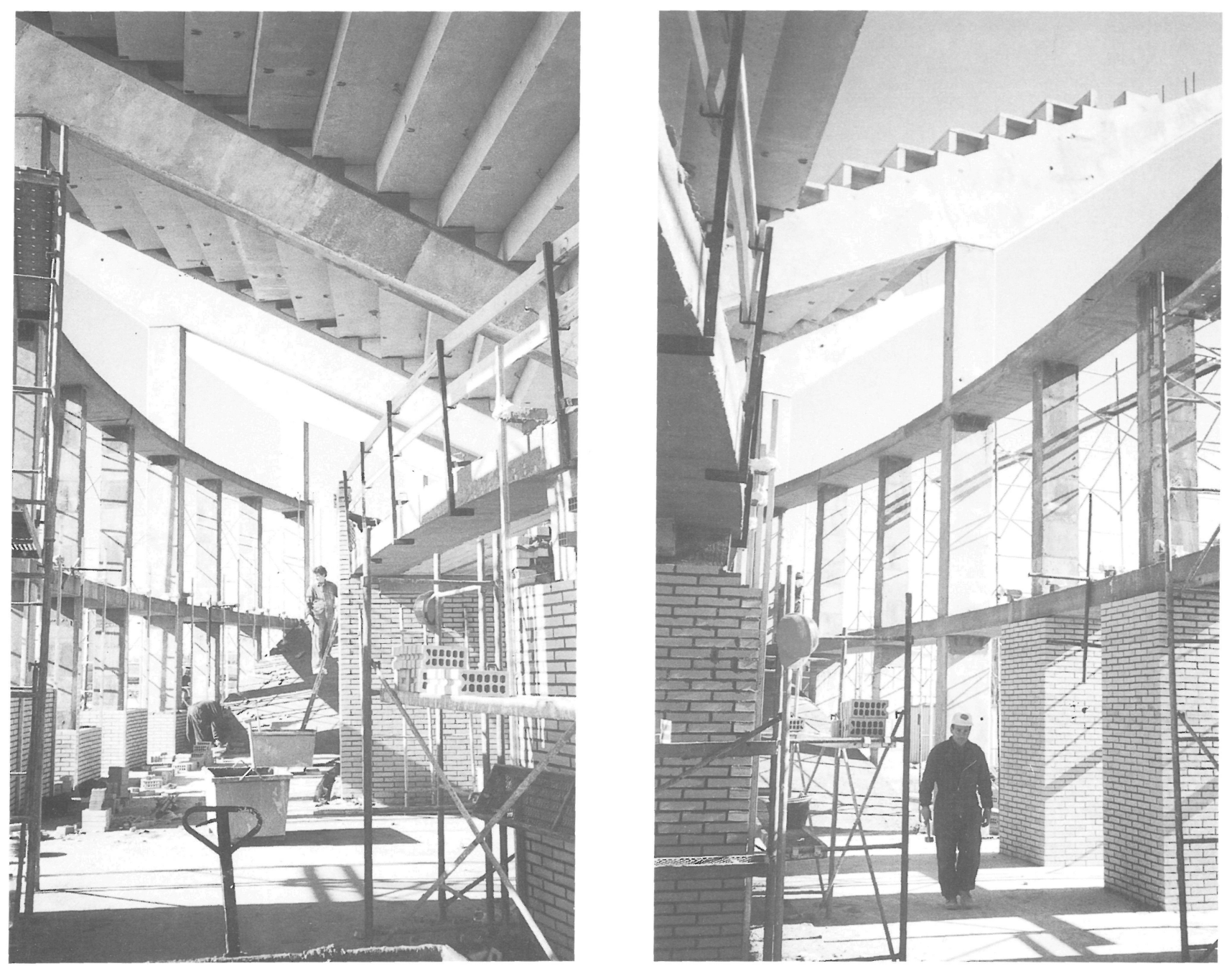

Pasillo de distribución.

tradición, pero su utilización, en forma de muros de doble hoja, atado, con armaduras de acero e hiladas de fábrica, a la vez que la continuidad del muro curvo, cortado por el plano inclinado que configura la plaza, le da un talante inequívocamente actual. Este material contrasta con el hormigón prefabricado visto que se utiliza para toda la estructura que, como hemos descrito anteriormente, configura gran parte del edificio y parece asentarse en los citados muros de ladrillo. Estos dos materiales configuran la imagen lejana de la plaza. En el detalle cobra protagonismo la madera de pino, tratada contra la humedad y el fuego y, por supuesto, pintada con el rojo característico de toda la vida. Utilización obligada por ser éste, ciertamente, el material que mejor sirve para la protección de las personas y de los animales, éstos de sus propias embestidas. Hay incrustaciones de madera de castaño en los puntos en los que se necesitaba mayor resistencia.

La cerrajería está configurada por perfiles de acero laminado comerciales, cortados de tal manera que resuelven cualquier detalle constructivo, dándole el aspecto rudo y fuerte que necesitan este tipo de edificios. Surgieron dificultades, casi anecdóticas, como determinar la fuerza que podía llegar a ejercer un toro de $500 \mathrm{~kg}$ contra las tablas de la carpintería, con el fin de determinar los perfiles que la sujetaban. Reconozco que este caso se resolvió, aparte de los cálculos inconsistentes, aplicando el puro saber popular que, por qué no, un arquitecto español también tiene.

Anécdotas aparte, los elementos finales, fundamentalmente carpinterías y cerrajerías, se diseñaron desde el escrupuloso respeto al ambiente tradicional de una plaza de toros, pero aportando soluciones constructivas evolucionadas.

El resultado final del proceso descrito es un edificio que, sin renunciar a un carácter que responde a una tradición fuertemente consolidada en grandes sectores de la población, resuelve los nuevos condicionantes que impone la sociedad, con una arquitectura plenamente contemporánea. En ese sentido, creemos que responde a nuestros objetivos iniciales, de conjugar la Evolución con el respeto a la Tradición, abriendo un camino en un momento en el que la reconversión de los espacios, tradicionalmente destinados a los toros, parece irreversible. 


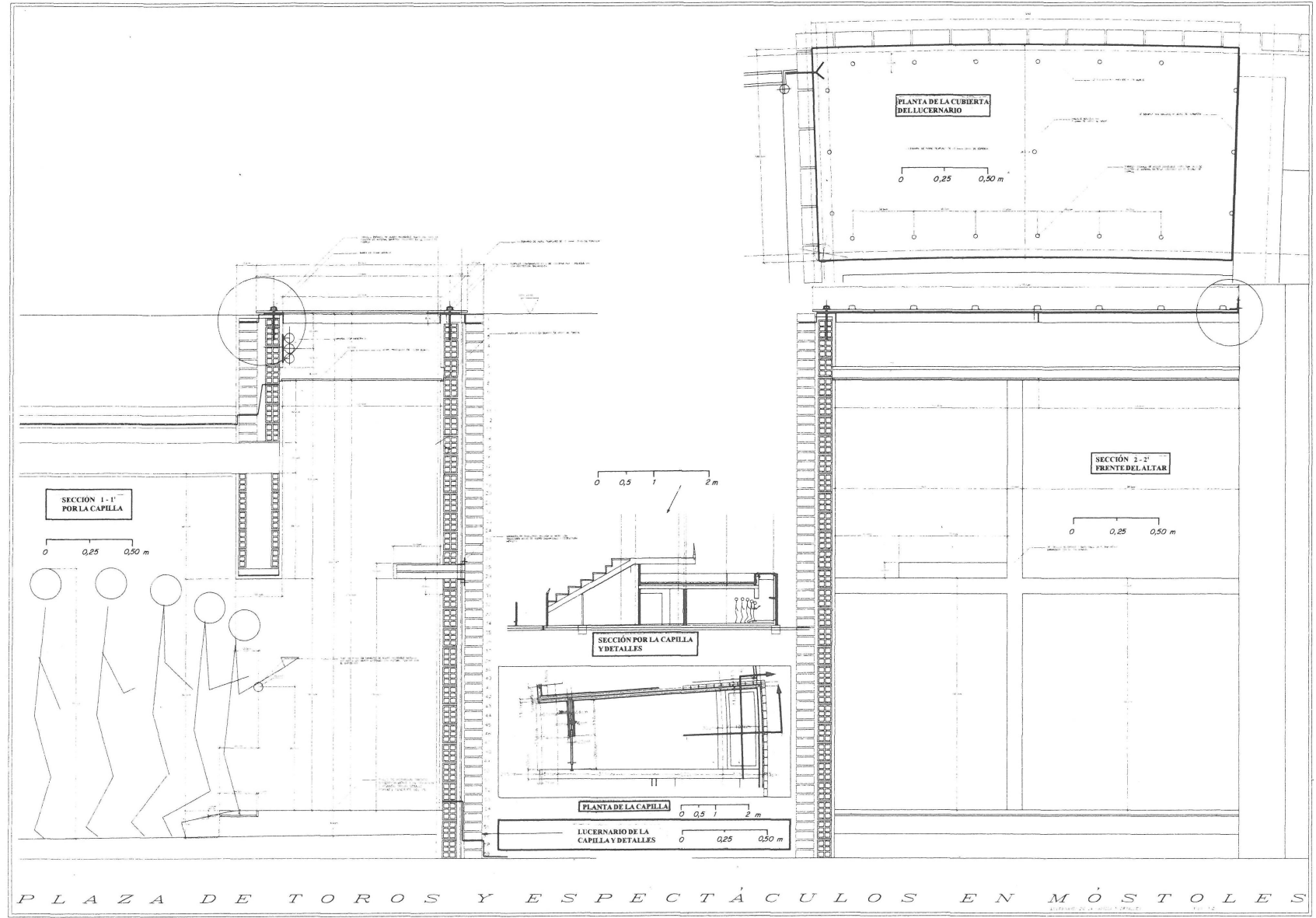

Detalle de la capilla.

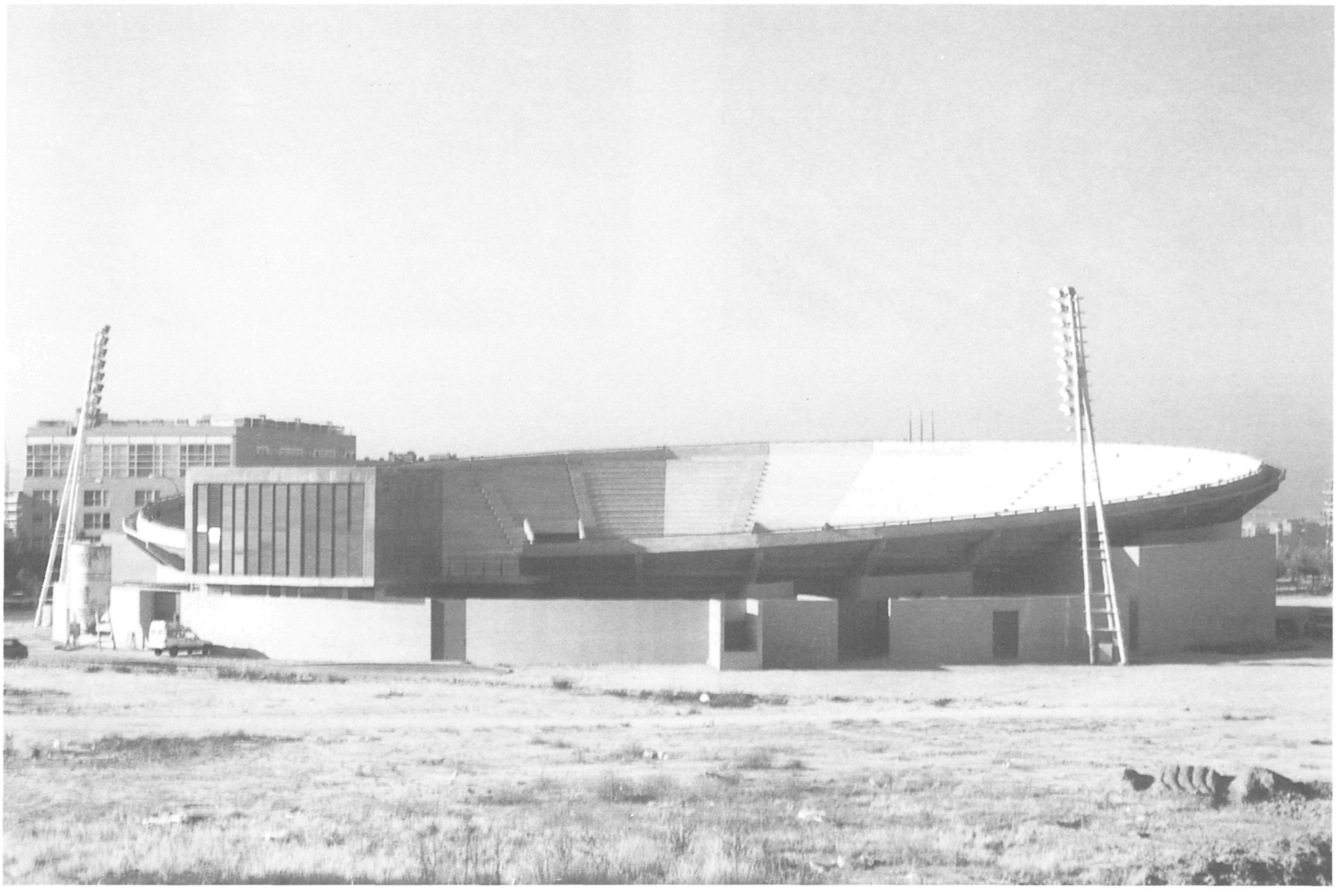

Vista desde el escenario. 

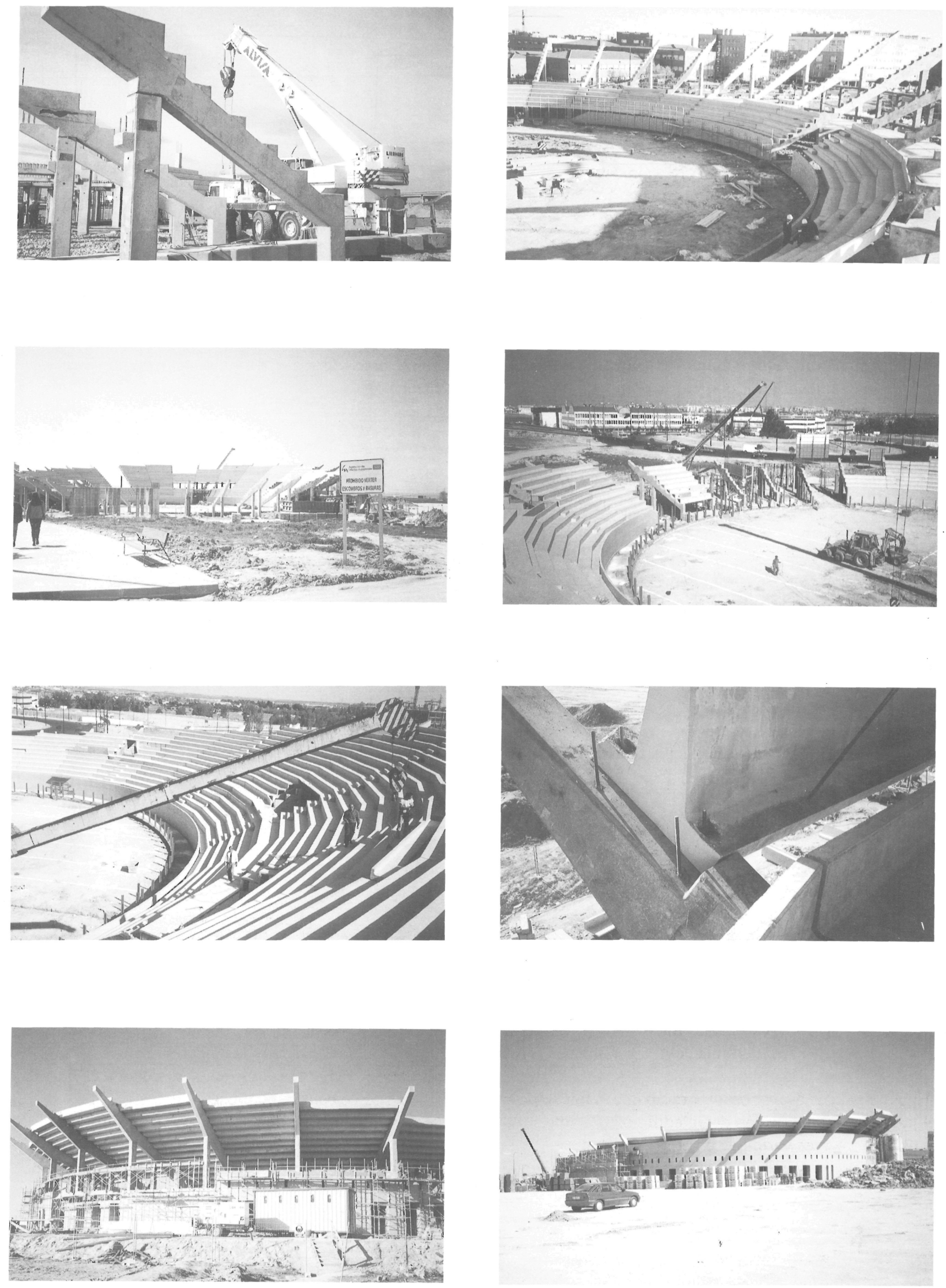

Distintas fases de la construcción. 